\title{
Flora das cangas da Serra dos Carajás, Pará, Brasil: Begoniaceae
}

\author{
Flora of the cangas of the Serra dos Carajás, Pará, Brazil: Begoniaceae
}

Ludovic Jean Charles Kollmann ${ }^{1,2}$

\begin{abstract}
Resumo
Este estudo apresenta as espécies de Begoniaceae registradas para as cangas da Serra dos Carajás, estado do Pará, com descrições, ilustrações e comentários morfológicos de cada espécie. São registradas até o momento quatro espécies de Begonia nas cangas da Serra dos Carajás: Begonia guaduensis, B. humilis, B. saxicola e B. wollnyi.
\end{abstract}

Palavras-chave: Begonia, FLONA Carajás, flora, taxonomia.

\begin{abstract}
This study encompasses the species of Begoniaceae registered for the cangas of Serra dos Carajás, Pará state with descriptions, illustrations and morphological comments of the species are provided. At the moment, four species of Begonia in the cangas of The Serra dos Carajás are registered: Begonia guaduensis, B. humilis, B. saxicola e B. wollnyi.
\end{abstract}

Key words: Begonia, FLONA Carajás, flora, taxonomy.

\section{Begoniaceae}

Begoniaceae C.Agardh, com cerca de 1400 espécies, é representada por dois gêneros: Hillebrandia Oliv., com uma espécie das ilhas Havaianas e Begonia L., que é pantropical, (Clement et al. 2004) mas ausente na Austrália (Tebbitt 2005). No Brasil ocorrem aproximadamente 213 espécies de Begonia, sendo 186 endêmicas, encontradas em quase todas as formações vegetais, entretanto, são mais frequentes na Serra do Mar, na região da Costa Atlântica onde são encontradas 180 espécies (BFG 2015), das quais 177 tem ocorrência restrita nesse domínio. Na Serra do Carajás foram registradas quatro espécies de Begonia, uma em formações rupestre e mata de canga e três em floresta de canga.

\section{Begonia L.}

Ervas, trepadeiras ou subarbustos, terrestres, epífitas ou hemiepífitas, glabras a pilosas. Caules carnosos, eretos, decumbentes ou prostrados, com entrenós distintos. Estípulas persistentes ou decíduas, eretas ou reflexas. Limbo basifixo ou peltado, simétrico ou não, nervação actinódroma ou craspedódroma. Cimeiras axilares de dicásio ou tirso, com ramos de 1-8 ordem, paucifloras ou multifloras, raro uniflora. Flores alvas, rosas ou vermelhas, pediceladas; as estaminadas com 2 ou 4 tépalas livres, anteras amarelas, rimosas ou poricidas, geralmente oblongas; flores pistiladas nas últimas ramificações, com (3-)5(-6) tépalas livres, as externas maiores, estiletes frequentemente 3, 2 ou 3 vezes partidos, papilas estigmáticas em faixas ou revestindo totalmente os ramos do estilete, ovário ínfero, 2-3-locular, raro 4-locular, frequentemente 3-alado, raro 4-alado, placentação axilar, placenta inteira ou bipartida, neste último caso, óvulos dispostos nas faces externas ou em ambas as faces das lamelas. Cápsula loculícida, rompimento irregular da parede, geralmente 3 alas, alas iguais a desiguais, raro ausentes. Sementes geralmente oblongas.

\footnotetext{
Instituto Nacional da Mata Atlântica/Museu de Biologia Prof. Mello Leitão, Av. Jose Ruschi 4, 29650-000, Santa Teresa, ES, Brasil.

${ }^{2}$ Autor para correspondência: ludokoll@yahoo.com.br
} 
Chave de identificação das espécies de Begonia das cangas da Serra dos Carajás

1. Plantas herbáceas anuais, margem da folha duplamente serrada...........................1.1 Begonia humilis

1'. Plantas subarbustivas a arbustiva perenes, margem da folha serrilhada a serrada

2. Lâminas lobadas com máculas.................................................................... 1.2 Begonia wollnyi

2'. Lâminas inteiras sem máculas

3. Estípulas persistentes, flores de $1-1,5 \mathrm{~cm}$ de diâmetro.

1.3 Begonia saxicola

3'. Estípulas decíduas, flores de 3-4 cm de diâmetro. 1.4 Begonia guaduensis

1.1. Begonia guaduensis Kunth, Nov. Gen. Sp. (quarto ed.) 7: 178-179. $1825 . \quad$ Fig. 1a-j

Arbusto perene, escandente 1-2 m alt., glabro, entrenós $0,7-5,2 \mathrm{~cm}$ compr., estípulas decíduas, $0,2-0,5 \mathrm{~cm}$ compr., pecíolo de $0,2-0,7 \mathrm{~cm}$ de compr., lâminas inteiras, transversalmente elípticas, 3,5-6,2 $\times 1,5-2,1 \mathrm{~cm}$, ápice acuminado, base assimétrica, margem serrilhada a serrada, ciliada, venação craspedródoma; cimeiras 12-23 cm compr., brácteas 5-9 × 3-6 mm, obovadas, ápice obtuso, flores de 3-4 cm de diâmetro, flores estaminadas sépalas 2 , lanceoladas a oblongas, $1,5-1,6 \times 0,8-1 \mathrm{~cm}$, ápice agudo a obtuso, pétalas 2 , elípticas, $1-1,1 \times$ $0,2-0,3 \mathrm{~cm}$, ápice agudo; flores pistiladas prófilos 2 , inseridos a baixo do hipanto, ovados, 5-7 × 3-4 mm, tépalas 5, desiguais, 7-11,5 × 3-7 mm, elípticas, ápice agudo a obtuso; cápsulas 1,3-1,5 × 0,6-0,8 cm, placenta bipartida, uma ala maior reta a ascendente e 2 muito menores, arredondadas.

Material examinado: Parauapebas, N3, 6 02 ' $33^{\prime \prime} \mathrm{S}$ $50^{\circ} 13^{\prime} 10^{\prime}$ 'W, 22.VI.2012, fl. e fr., L.V.C. Silva et al. 1299 (BHCB); N4-WS, 604'33"S 50¹1'1'"W, 679m, 23.IV.2012, fl., A.J. Arruda et al. 1066 (BHCB, MG).

Subarbusto a arbusto de crescimento ereto se apoiando na vegetação próxima ao decorrer de seu crescimento. Folhas elípticas de 3 a $6 \mathrm{~cm}$ de comprimento. Flores grandes de aproximadamente $3 \mathrm{~cm}$ de diâmetro. Pode ser diferenciada de Begonia saxicola A.DC., com qual é confundida no campo e no material de herbário, pelas estípulas decíduas (vs. permanentes) e flores de 3-4 cm de diâmetro (vs. 1-1,5 cm).

Begonia guaduensis ocorre na Costa Rica, Panamá, Colômbia, Equador, Venezuela e Brasil. No Brasil na região amazônica: Amazônia, Mato Grosso, Rondônia, Roraima e Pará. Na Serra dos Carajás foi coletada na Serra Norte: N3 e N4. É encontrada geralmente em cima de afloramento rochoso na borda ou dentro da mata de canga.

1.2. Begonia humilis Dryand, Hort. Kew., $3: 353$, 1789.

Fig. 1k-y

Ervas anuais, $3-38 \mathrm{~cm}$ alt., glabras a pilosas, entrenós $0,5-6,6 \mathrm{~cm}$ compr., glabros a pilosos, estípulas persistentes 3-4,4×0,6-2,4 mm, margens inteiras a laciniadas, pecíolo $0,2-3 \mathrm{~cm}$ de compr., lâminas inteiras, transversalmente elípticas $0,9-7$ $\times 0,4-2,6 \mathrm{~cm}$, ápice acuminado, base cordada, margem duplamente serrada, ciliada, face adaxial pilosa, tricomas simples, face abaxial glabra, venação actinódroma; cimeiras $1-7,5 \mathrm{~cm}$ compr., flores estaminadas sépalas 2, ovadas a reniformes, 2,5-4,5 × 2-3,3 mm, ápice arredondado, estames em uma coluna; flores pistiladas prófilos 3 , inseridos abaixo do hipanto, ovados, 2,7-6 $\times$ 2,5-4,3 $\mathrm{mm}$, lanceoladas a triangulares, margens fimbriadas, tépalas 5, iguais entre si, 1,5-3 $\times$ 0,9-1,3 mm, elípticas a obovadas, ápice arredondo a obtuso, margem inteira; cápsulas 5-14 × 4-20 $\mathrm{mm}$, placenta inteira a bipartida, uma ala maior reta a ascendente, ápice obtuso e 2 menores, arredondadas.

Material examinado: Canaã dos Carajás, $6^{\circ} 24^{\prime} 00^{\prime \prime}$, $50^{\circ} 18^{\prime} 56^{\prime}$ "W, 12.V.2014, fr., R.S. Santos et al. 186 (MG); 6²4'29,6”' 50¹4'2252,35”'W, 270 m, 27.IV.2010, fl. e fr., F.D. Gontijo et al. 160 (BHCB); Racha Placa, ADA Usina S11, 6²4'33"'S, 50¹4'50”'W, 275 m, 28.I.2012, fl., L.F.A. Paula de et al. 479 (BHCB); Serra Sul, ao longo da estrada S11D até S11A, 6 ${ }^{\circ} 22^{\prime} 17^{\prime \prime}$,, $50^{\circ} 23^{\prime} 04^{\prime \prime} \mathrm{W}$, 23.III.2015, fl. e fr., L.C. Lobato et al. 4416 (MG); Serra Sul, corpo B, $6^{\circ} 22^{\prime} 43,2^{\prime}$ 'S, 50²2' 45,31 ”'W, 667 m, 16.II.2010, fl. e fr., F.D. Gontijo 90 (BHCB); S11-C, 622'22'"S, 50²3'30'W, fl., 22.03.2012, P.B. Meyer 1157 (BHCB); Serra Sul, corpo C, 6²3'54,33's, $50^{\circ} 21^{\prime} 59,54$ 'W, 729 m, 19.II.2010, fl., F.D. Gontijo 130 (BHCB); Serra Sul, Platô S11D, Capão, 6²4'00"S, $50^{\circ} 18 ' 56^{\prime}$ 'W, 12.V.2014, fr., R.S. Santos et al. 183 (MG); S11-D, 6²3'32”'S, 50²2'18”'W, 20.III.2012, fl., A.J. Arruda et al. 721 (BHCB); Serra Sul, corpo D, 623'55"S, 50¹6'39"W, 700 m, 17.III.2009, fl., P.L. Viana. et al. 4105 (BHCB);Tarzan, 6¹9'29's, $50^{\circ} 07$ '22”'W, $770 \mathrm{~m}, 20 . V I .2012$, fr., L.V.C. Silva et al. 1242 (BHCB); Serra do rabo, $6^{\circ} 17^{\prime} 03$ ' $\mathrm{S}, 49^{\circ} 55^{\prime} 02^{\prime}$ 'W, 20.XII.2010, fl., N.F.O. Mota et al. 2021 (BHCB); Canaã dos Carajás, [Marabá], Serra Sul, 16.IV.1986, fr., R. Secco et al. 716 (MG); Parauapebas, Serra Norte, N1, 601'38'S, 50¹7'29' W, 700 m, 12.III.2009, fl., P.L. Viana. et al. 3792 (BHCB); N2, 603'27'S, 50¹4'49”'W, 721 m, 19.IV.2012, fl. e fr., A.J. Arruda et al. 950 (BHCB); N3, 602'32”'S, 50¹3'08”'W, 608 


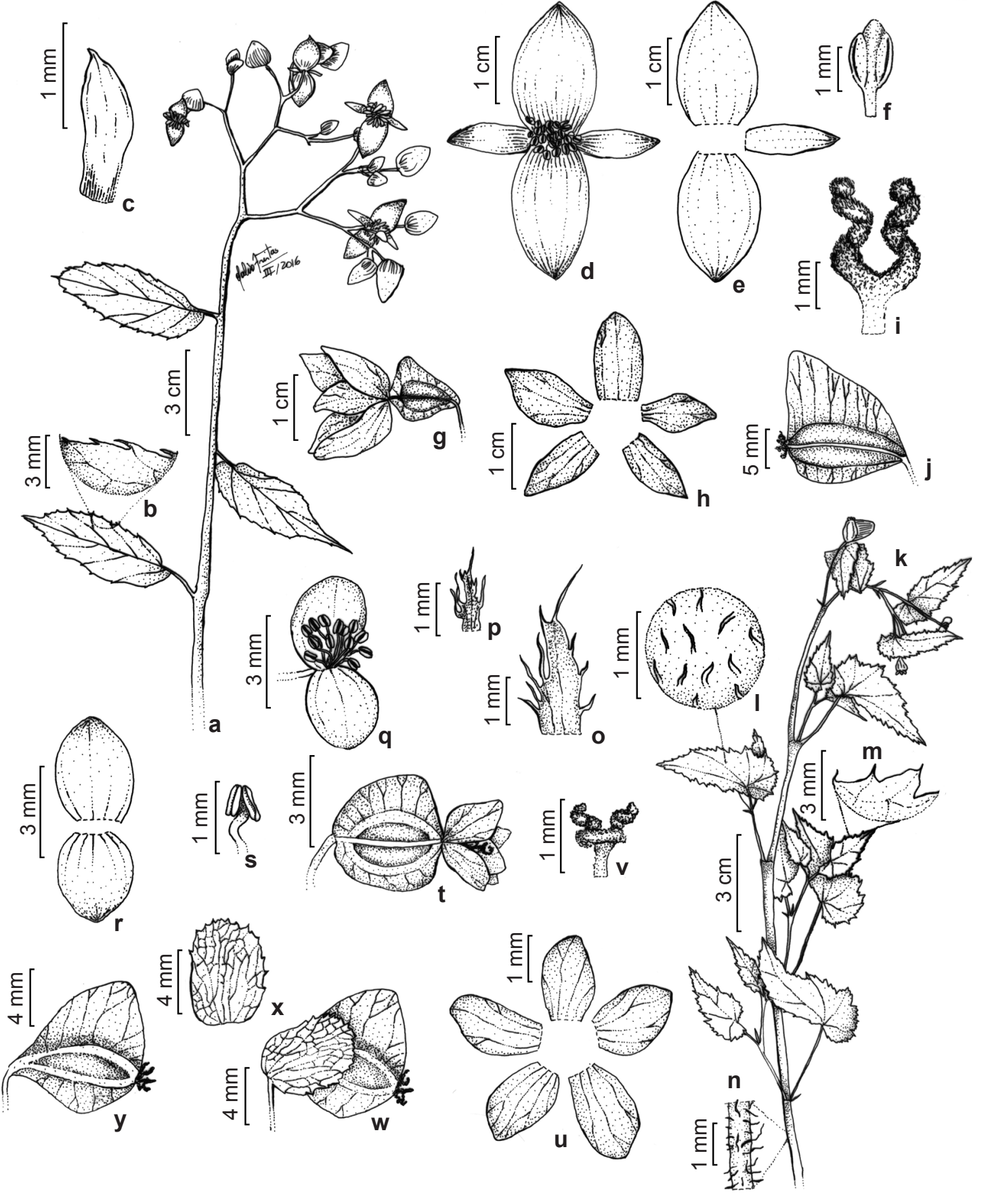

Figura 1 - a-j. Begonia guaduensis - a. hábito; b. detalhe da margem da lâmina; c. estípula; d. flor masculina; e. sépalas (vertical), pétala (horizontal); f. estame; g. flor feminina com fruto imaturo; h. tépalas da flor feminina; i. estigma; j. fruto. k-y. Begonia humilis - k. hábito; 1. pilosidade na face adaxial da lâmina; m. detalhe da margem da lâmina; n. pilosidade do caule; o. estípula; p. bráctea; q. flor masculina com fruto imaturo; r. sépalas; s. estame; t. flor feminina; u. tépalas da flor feminina; v. estigma; w. fruto com prófilo; x. prófilo; y. fruto. (a-i. A.J. Arruda 1066; j. L.V.C. Silva 1299; k-y. P.B. Meyer 1157).

Figure 1 - a-j. Begonia guaduensis - a. habit; b. detail of lamina margin; c. stipule; d. mal flower; e. sepals (vertical), petal (horizontal); f. stamen; g. female flower with immature fruit; h. tepals of female flower; i. stigma; j. fruit. k-y. Begonia humilis - k. habit; 1. hairiness of adaxial face; m. detail of lamina margin; $n$. hairiness of stem; o. stipule; $p$. bract; q. mal flower; r. sepals; s. stamen; t. female flower; u. tepals of female flower; v. stigma; w. fruit with profile; x. profile; y. fruit. (a-i. A.J. Arruda 1066; j. L.V.C. Silva 1299; k-y. P.B. Meyer 1157). 
m, 22.VI.2012, fr., L.V.C. Silva et al. 1296 (BHCB); N4, 08.III.2010, fl., L.C.B. Lobato et al. 3886 (MG); N4WS, 604'22"S, 50॰11'42"'W, 505 m, 24.III.2012, fl., P.B. Meyer 197 (BHCB); Platô AMZA, camp. N-5, 64'S 5008'W, 700-750 m, 12.V.1982, fr., C.R. Sperling et al. 5591 (MG, NY, MO); Parauapebas, [Marabá] Serra dos Carajás, $6^{\circ} 00^{\prime} \mathrm{S}, 50^{\circ} 18^{\prime} \mathrm{W}, 700 \mathrm{~m}, 24$.V.1969, fr., $P$. Calvacante 2156 (MG); Capão, 04.VI.1983, fr., M.F.F. da Silva et al. 1481 (MG); Serra Norte N1, área do túnel 6, 21.IV.1970, fr., M. Silva 2672 (MG); N-4, mina piloto, 700-750 m, 14.III.1984, fr., A.S.L da Silva et al. 1772 (MG); Serra Norte, arredores N5, 12.V.1982, fr., R. Secco et al. 125 (MG).

Planta anual, vegetando principalmente na época úmida podendo ser encontrada às vezes abundantemente em diferentes locais como beira de estrada, campos rupestres, beira e interior da mata em cima de canga. Planta facilmente reconhecível pelo porte herbáceo com caule não lenhoso, folhas elípticas a ovadas de base cordada, margens profundamente biserradas. Inflorescência do tamanho da folha ou ultrapassando levemente com poucas flores, mas muitas inflorescências no indivíduo.

Begonia humilis ocorre do México até a Guiana Francesa. No Brasil, região amazônica e nordeste: Acre, Amazônia, Pará, Roraima, Alagoas, Ceara e Pernambuco. Na Serra dos Carajás há registros na Serra Norte: N1, N2, N4 e N5, Serra Sul: S11-A, S11-B, S11-C e S11-D. Begonia humilis é encontrada geralmente em cima de afloramento rochoso fora ou dentro da mata de canga e áreas antropizadas como beira de estrada.

1.3. Begonia saxicola A.DC., Ann. Sci. Nat.; Bot., sér. 4 11: 144. 1859.

Fig. $2 \mathrm{a}-\mathrm{j}$

Arbusto perene, escandente 0,7-1,6 m

alt., piloso, entrenós $3-13 \mathrm{~cm}$ compr., estípulas persistentes, $0,2-1,5 \mathrm{~cm}$ compr., triangulares, ápice agudo, margem inteira, pecíolo $0,8-2 \mathrm{~cm}$ de compr., lâminas inteiras, elípticas 4-9,5 × 1,5-5 cm, ápice agudo, base assimétrica, margem serrilhada a serrada, ciliada, face adaxial glabra, face abaxial pilosa, tricomas simples, venação craspedódroma; cimeiras 5-22 cm compr., brácteas 1-2 mm compr., triangulares, flores de $1-1,5 \mathrm{~cm}$ de diâmetro, flores estaminadas sépalas 2, 5,5-7,5 ×6,5-7 mm, reniformes, ápice arredondado, pétalas 2, 3-4× $1-3,3 \mathrm{~mm}$, elípticas, ápice obtuso; flores pistiladas prófilos 2 , inseridos no pedicelo, triangulares, 0,5-1,6 mm compr., tépalas 5, desiguais, $2-8 \times$ 1-6 mm, ovadas a elípticas a obovadas, capsulas $0,6-1,1 \times 0,4-0,5 \mathrm{~cm}$, placenta bipartida, uma ala maior ascendente e 2 menores, redondas.
Material examinado: Canaã dos Carajás, Serra Sul, 16.IV.1986, fl., R. Secco et al. 710 (MG); Serra Sul, 6²4'00"S, 50¹8'56"W, 12.V.2014, fl., R.S. Santos et al. 185 (MG); Serra Sul, 6²3'53'S, 50²0'57'W, 14.IV.2015, fl. e fr., L.M.M. Careira et al. 3369 (MG); S11-A, 6²0'00'S, 50²7'90”'W, 673 m, 21.III.2012, fl., P.B. Meyer et al. 1138 (BHCB); S:SW do corpo A, $6^{\circ} 19^{\prime} 43.13$ 'S, 50²7'17.68'”, $741 \mathrm{~m}, 15 . I I .2010$, fr., F.D. Gontijo 83 (BHCB); S11-C, 6²4'0"'S, 50²3' 1' W, 663 m, 29.XII.2012, fr., L.V.C Silva et al. 1167 (BHCB); 6²2'22''S, 50²3'30'”W, $723 \mathrm{~m}, 22$. III.2012, fl., P.B. Meyer et al. 1155 (BHCB); corpo C, 6²3'02”'S, 50²3'43”'W, 28.VI.2010, fl., T.F. Almeida et al. 2412 (BHCB); corpo D, 10.X.2008, fr., L.V. Costa et al. 685 (BHCB); ponto 79, 10.X.2008, fr., L.V. Costa et al. 526 (BHCB); Serra Sul, corpo D, 6²3'08'S, $50^{\circ} 23$ '5"W, 16.III.2009, fl., V.T. Giorni et al. 183 (BHCB); Parauapebas, estrada serra sul, 6¹7'02'S, 50²0'13'”, 02.VI.2010, fl., A.J.Arruda 335 (BHCB); Serra Norte, $6^{\circ} \mathrm{S}, 50^{\circ} 15^{\prime} \mathrm{W}, 16 . X .1977$, fr., C.C. Berg \& A.J. Henderson 567 (MG); Serra dos Carajás, N-4, 19.III.1984, fl., A.S.L. da Silva et al. 1913 (MG); N4-WS, 604'33"S, 50¹1'1'W, 23.IV.2012, fl., A.J. Arruda et al. 1067 (BHCB); Serra Norte, platô N8, 6¹0'01'S, 5009'29'W, 18.III.2015, fl., L.C. Lobato et al. 3369 (MG); Parauapebas, [Marabá] N-4, 29.III.1977, fl., M.G. Silva \& R. Bahia 2934 (MG); Serra Norte, N4, 12.V.1982, fr., R. Secco et al. 411 (MG); N5, 22.IV.1986, fl. e fr., R.S. Seco \& R. Bahia 732 (MG).

Subarbusto a arbusto de crescimento ereto se apoiando na vegetação próxima ao decorrer de seu crescimento. Folhas elípticas de 4 a 9,5 cm de comprimento. Flores pequenas de aproximadamente $1,5 \mathrm{~cm}$ de diâmetro. Pode ser diferenciada de Begonia guaduensis, com qual é confundida no campo e no material de herbário, pelas estípulas permanentes (vs. decíduas) e flores de $1-1,5 \mathrm{~cm}$ de diâmetro (vs. $3-4 \mathrm{~cm}$ ).

Begonia saxicola é encontrada na região amazônica e nordeste, nos estados do Acre, Alagoas, Bahia, Ceará, Pará, Paraíba e Pernambuco. $\mathrm{Na}$ Serra dos Carajás foi registrada na Serra Norte: N4, N5 e N8, Serra Sul: S11-A, S11-C e S11-D. É encontrada geralmente em cima de afloramento rochoso na borda ou dentro da mata de canga.

1.4. Begonia wollnyi Herzog, Repert. Spec. Nov. Regni Veg. 7: 63. 1909.

Fig. 2k-u

Subarbusto perene, 0,25-0,5 $\mathrm{m}$ alt., piloso, tricomas simples, túberas $1,5-2 \times 2-3 \mathrm{~cm}$, entrenós 0,3-3 cm compr., estípulas persistentes, triangulares a ovadas, ápice agudo margem inteira, 0,8-1 × 0,3$0,5 \mathrm{~cm}$, folhas com pecíolo de $7-12 \mathrm{~cm}$ de compr., lâminas palmatifendas, ovadas 8-20 × 11-26 cm, 6-7 lobos, ápice dos lobos acuminados, base 


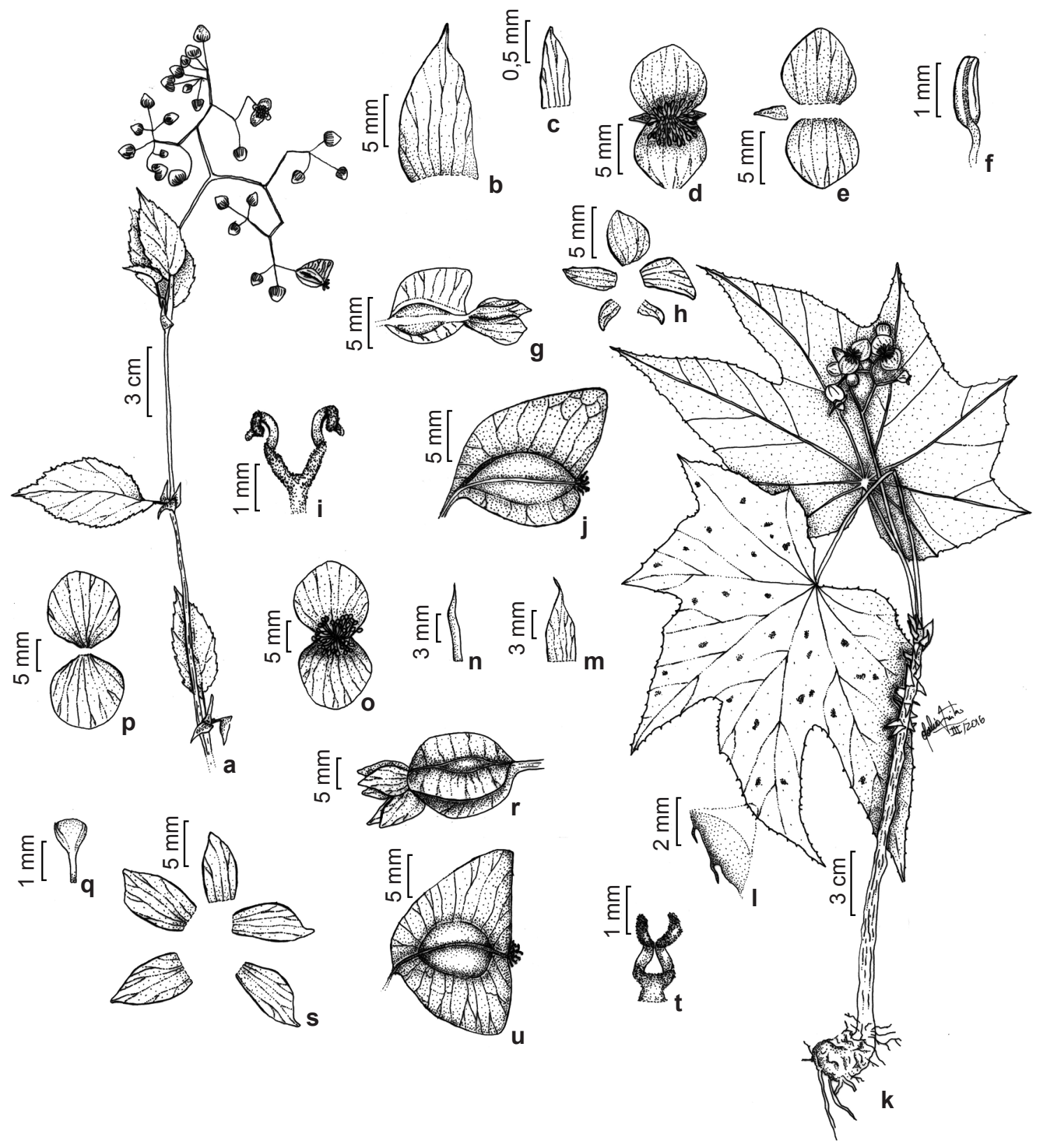

Figura 2 - a-j. Begonia saxicola - a. hábito; b. estípula; c. bráctea; d flor masculina; e. sépalas (vertical), pétala (horizontal); f. estame; g. flor feminina com fruto imaturo; h. tépalas da flor feminina; i. estigma; j. fruto. k-u. Begonia wollnyi-k. hábito; 1 . detalhe da margem da lâmina; m. estípula; n. bráctea; o. flor masculina; p. sépalas; q. estame; r. flor feminina com fruto imaturo; s. tépalas da flor feminina; t. estigma; u. fruto (a-f. L.C. Lobato 4340; g-i. R.S. Santos 185; j. L.V. Costa 526; k-n, u. L.F.A. de Paula 450; o-q. L.V.C. Silva 1319; r-t. R.S. Santos 184)

Figura 2 - a-j. Begonia saxicola - a. habit; b. stipule; c. bract; d mal flower; e. sepals (vertical), petal (horizontal); f. stamen; g. female flower with immature fruit; h. tepals of female flower; i. stigma; j. fruit. k-u. Begonia wollnyi -k. habit; 1 . detail of lamina margin; m. stipule; n. bract; o. mal flower; p. sepals; q. stamen; r. female flower with immature fruit; s. tepals of female flower; t. stigma; u. fruit. (a-f. L.C. Lobato 4340; g-i. R.S. Santos 185; j. L.V. Costa 526; k-n, u. L.F.A. de Paula 450; o-q. L.V.C. Silva 1319; r-t. R.S. Santos 184) 
cordada, margem serrilhada, ciliada, face adaxial ligeiramente pilosa, com máculas esbranquiçadas, face abaxial glabra, venação actinódroma, 6-7 nervuras na base; cimeiras 5,5-21 cm compr., 2-3 dicotomas, brácteas 1-2 mm compr., flores estaminadas, sépalas 2, 0,7-0,9 ×0,9-1 cm, ovadas, ápice arredondado; flores pistiladas, prófilos 2 , ca. $3 \mathrm{~mm}$ compr., decíduos, tépalas 5 , iguais entre si $3,5-8,5 \times 2-5 \mathrm{~mm}$, ovadas, ápice obtuso a agudo; cápsulas com alas 1,5-2,3 × 2-2,6 cm, placenta bipartida, uma ala maior arredondada a aguda, reta a ascendente e 2 menores, arredondadas.

Material examinado: 6"W, 6.X.2009, fr., V.T. Giorni et al. 2427 (BHCB); S11-D, 6²3’31,3”'S, 50¹9'9,06”'W, 22.VII.2012 fl. e fr., A.J. Arruda et al. 1217 (BHCB); S11-D, 623'31'S, 50¹9'9”W, 23.V.2012, fl., A.J. Arruda et al. 1176 (BHCB, MG); S11-D, 6 23'29"S, 50¹9'04”'W, 26.I.2012, fr., L.F.A. de Paula et al. 450 (BHCB); Serra Sul, S11-D, 624'00”S, 50¹8'56"W, 12.VI,2014, fr., R.S. Santos et al. 184 (MG); Subida da Cachoeira, 6²4'29,6”S, 50¹4'52,35”'W, 27.IV.2010, fr., F.D. Gontijo et al. 159 (BHCB); Parauapebas, Serra dos Carajás, Azul, campo de manganês abandonado, $6^{\circ} 06^{\prime}$ S, 50 17 'W, fl., 31.V.1982, fl., C.R. Sperling et al. 5903 (MG, MO); perto do campo da Serra Norte, $5^{\circ} 59^{\prime} \mathrm{S}$, 50²8'W, 8.XII.1981, fr., D.C. Daly et al. 1861 (MG, NHNH, NY); N7, 609'10”'S, 50¹0'42”'W, 24.VI.2012, fl., L.V.C Silva et al. 1319 (BHCB, MG).

Subarbusto de caule lenhoso ereto. Planta facilmente reconhecível pelas folhas fortemente lobadas com máculas prateadas.
Begonia wollnyi ocorre na Bolívia e Brasil: Acre, Amazonas, Pará e Roraima. Na Serra dos Carajás foi coletada na Serra Norte: N7, Serra Sul: S11-A, S11-B e S11-D. Cresce em cima de afloramento rochoso nas matas de canga. Também foi encontrada em área de exploração de manganês na Serra do Carajás.

\section{Agradecimentos}

Ao Museu Paraense Emílio Goeldi e ao Instituto Tecnológico Vale, o apoio à elaboração deste trabalho. Ao Instituto Nacional da Mata Atlântica (INMA), Ministério da Ciência, Tecnologia e Inovação (MCTIC) e CNPq, a bolsa de Programa de Capacitação Institucional. Aos curadores dos herbários BHCB e MG por disponibilizar o material estudado.

\section{Referências}

BFG. 2015. Growing knowledge: an overview of seed plant diversity in Brazil. Rodriguésia 66: 1085-1113.

Clement, W.L.; Tebbit, M.C.; Forrest, L.L.; Blair, J.E.; Brouillet, L.; Erikson, T. \& Swensen, S.M. 2004. Phylogenetic position and biogeography of Hillebrandia sandwicensis (Begoniaceae): a rare Hawaiian relict. American Journal of Botany 91: 905-917.

Tebbit, M.C. 2005. Begonias: cultivation, identification, and natural history. Timber Press, Inc. Portland. 272p.

\footnotetext{
Lista de exsicatas

Almeida, T.F. 2412 (1.3), 2427 (1.4); Arruda, A.J. 335 (1.3), 721 (1.2), 950 (1.2), 1066 (1.1), 1067 (1.3), 1176 (1.4), 1217 (1.4); Berg, C.C. 567 (1.3); Careira, L.M.M. 3369 (1.3); Cavalcanti, P. 2156 (1.2); Costa, L.V. 526 (1.3), 685 (1.3); Daly, D.C. 1861 (1.4); Giorni, V.T. 183 (1.3), 2427 (1.4); Gontijo, F.D. 82 (1.4), 83 (1.3), 90 (1.2), 130 (1.2), 159 (1.4), 160 (1.2); Lobato, L.C. 3369 (1.3), 3886 (2), 4416 (1.2); Meyer, P.B. 197 (1.2), 1138 (1.3), 1155 (1.3), 1157 (1.2); Mota, N.F.O. 2021 (1.2); Paula, L.F.A. 450 (1.4), 479 (1.2); Santos, R.S. 183 (1.2), 184 (1.4), 185 (1.3), 186 (1.2); Secco, R.S. 41 (1.3), 711 (1.3), 716 (1.2), 732 (1.3); Silva, A.S.L da 1772 (1.2), 1913 (1.3); Silva, L.V.C. 1167 (1.3), 1242 (1.2), 1296 (1.2), 1299 (1.1), 1319 (1.4); Silva, M. 2672 (1.2); Silva, M.G. 2934 (1.3); Silva, M.F.F. da 1481 (1.2); Sperling, C.R. 5591 (1.2), 5903 (4); Viana, P.L. 3792 (1.2), 4105 (1.2).
} 